of many tried which shows a clear difference between healthy and 'deficient' soils.

It seems to follow that manganese is absorbed by the root without first passing into the soil solution as the ion $\mathrm{Mn}++$; the absorption might be either direct as colloidal $\mathrm{MnO}_{2}$, or by reduction of $\mathrm{MnO}_{2}$ at the root-soil interface-a reduction which becomes steadily more difficult as the $p \mathrm{H}$ rises, until only very active $\mathrm{MnO}_{2}$ can take part in the reaction.

This same test may be expected to show whether it is dangerous to lime on acid soil ; trouble may follow if the total manganese dissolved by ammonium acetate and quinol at $p \mathrm{H} 7$ does not exceed about 15 parts per million of soil. This suggestion cannot be tested here at present, since manganese deficiency following overliming has as yet been proved on only one Australian soil, itself abnormal. It is thought also that light may be thrown on the state of manganese in the soil horizons by leaching with buffer solutions (such as ammonium acetate) of varying $p \mathrm{H}$ values, with or without the addition of reducing agents (such as quinol) capable of bringing about definite $r \mathrm{H}$ values. The details of the work will appear in the Proceedings of the Royal Society of Victoria.

G. W. LEeper.

School of Agriculture,

University,

Melbourne, N.3.

Oct. 18.

\section{Publication of Nomina Nuda}

DR. VAN DER HORST'S communication in NATURE of December 1, p. 852, is of much interest in giving exact information with regard to the conformation of the burrow of an Enteropneust-a subject about which little is known. Without wishing to detract in any way from the value of his note, I find it necessary to criticise one part of it. It is well known to workers in systematic zoology that great inconvenience is caused by the publication of nomina nuda or names which are unaccompanied by diagnoses of the new species to which they refer. The binomial designation given to the new species of which a description is to be published later by one of Dr. van der Horst's students is presumably of this character. It may indeed prove to be the case that the form of the burrow is by itself distinctive of the new species; and it might perhaps be argued that since this had been described and figured the name is valid. There are probably few zoologists, however, who would recognise a species of which the type-specimen was a mass of sand and mud containing no part of the animal itself except some of its slime.

The trouble given to taxonomists by introductions of this kind is very real. It is often necessary to waste time and print by explaining why the date of the first mention of a name cannot be accepted as the valid date. Many instances are known in which the nomen nudum has not been reprinted, but it remains to encumber the literature. The most troublesome cases, however, are probably those in which controversy is possible on the question whether a name is a nomen nudum or not. I feel confident that the great majority of systematists would agree with me in requesting the editor of NATURE to delete all such names from future communications sent for publication in his pages.

SIDNEY F. HARMER.

Melbourn, Cambs.

Dec. 1.

\section{Design of Theodolite Axes}

IN NATURE of September 15 a letter from Prof. A. F. C. Pollard points out that the cylindrical bearings adopted for the vertical axis of the Wild precision theodolite leads to a systematic error of the "order of two to four seconds" in the horizontal angles. We, in Egypt, have not had much experience with theodolites of the Wild design, but we have found that the substitution of cylindrical for the old $Y$-bearings for the telescope (trunnion) axis of English theodolites has not been found to be an improvement but very much the reverse. Whilst the instrument is new, the errors arising from this fault in design do not obtrude themselves, but as soon as the bearings become worn or one of the standards gets slightly bent, the telescope comes to rest in a different position every time it is raised or lowered in elevation. The error arising from this defect is of an 'accidental' nature and may, in an extreme case, amount to one minute.

Another defect in modern English design adopted by some firms is the tightening arrangement for taking up wear in the levelling screws. The old fashioned method of making a vertical radial cut along the centre of the trivet arm and placing a binding screw at its outer end was perfectly satisfactory and should never have been given up. The modern designs either do not work at all or they grip the screw at one point only instead of along the whole length of the thread.

In addition to these defects, we have had trouble owing to the poor optical qualities of the modern telescopes. Distant points which are easily sighted with an old-fashioned theodolite are invisible in a modern telescope. This is probably due to the adoption of the internal focusing arrangement. The introduction of an additional lens (or lenses) in the optical system cuts out light and impairs the definition.

Speaking generally, the so-called 'dust-proof' covers are a continual source of trouble. We have never found one which keeps out the dust. Also they make the theodolite much more difficult to keep in good order.

Leaving out of account modern instruments of the Wild type, the old Troughton and Simms six-inch theodolite of thirty years ago was the nearest approach to a perfect instrument for field triangulation that has yet been made. We have not found any modifica. tion in the design of this type of instrument which has not been found by experience to be a chango for the worse.

F. S. RICHARDS.

\section{Survey of Egypt,}

$$
\text { El-Giza. }
$$

Mr. F. S. RIChards's letter is an interesting corroboration of the points raised in my original communication and it is hoped that theodolite manufacturers will give the criticisms of Rannie and Dennis, as well as those of Richards, the attention they deserve.

Mr. Richards's remarks about levelling screws are important. In the paper by Rannie and Dennis, to which I have given the reference, it is recorded that badly designed levelling screws were a source of error in the readings of the instrument. In their case, changing the positions of the levelling screws and clamping them strained the alidade axis. Mr. Richards complains that in modern designs the screw-spindle of the levelling screw is imperfectly clamped.

These imperfections can be completely and readily 